\title{
Vertex reconstruction and tracking in the trigger algorithm for CMS
}

\author{
Marcin Konecki* \\ Institute of Experimental Physics, University of Warsaw, Poland \\ E-mail: marcin.koneckidcern.ch
}

Triggering in the high-rate environment of LHC is a challenging task. The Compact Muon Solenoid (CMS) experiment has developed a two-stage trigger system. The Level-1 Trigger is based on custom hardware devices while the High-Level Trigger (HLT) is using a farm of commercial processors.

One of the key aspects of HLT reconstruction is a fast and efficient track and vertex finding, in which CMS Pixel Detector, the innermost in the tracking system, is of primary importance. It provides precise measurements not only supporting the full track reconstruction, but also allowing the standalone reconstruction which is well suited for CMS online selection.

The track and vertex reconstruction algorithms for HLT are presented with a focus on fast pixel track reconstruction, primary-vertex finding and seed generation.

The 16th International Workshop on Vertex detectors

September 23-28 2007

Lake Placid, NY, USA

* On behalf of the CMS Collaboration 


\section{Introduction}

The Large Hadron Collider (LHC) is the next $p-p$ accelerator $^{1}$ at CERN. The main motivation [1] for building the LHC is to explore the energy region up to a few $\mathrm{TeV}$ range, in view of improving the understanding of the Electroweak Symmetry Breaking mechanism, and of searching for Supersymmetry and any kind of New Physics. Precise tests of the Standard Model are also important topics.

Since the cross section in some discovery channels can be very low, a large luminosity for LHC is mandatory. It is expected, that after the startup luminosity of $10^{32} \mathrm{~cm}^{-2} \mathrm{~s}^{-1}$ the luminosity will reach $2 \cdot 10^{33} \mathrm{~cm}^{-2} \mathrm{~s}^{-1}$ (so called low luminosity mode) and will be increased later to the designed luminosity of $10^{34} \mathrm{~cm}^{-2} \mathrm{~s}^{-1}$ (high luminosity mode).

The Compact Muon Solenoid (CMS), currently in commissioning phase, is a general purpose experiment for physics discoveries at the highest luminosities of LHC. The CMS design principles [2, 3] include efficient lepton and photon selection and measurements. These particles are very important for low statistics discovery physics. The event selection system has to be flexible to preserve signal signatures and efficiently reduce the background. In order to achieve that, events have to be partially reconstructed online, what makes the track reconstruction a key point of CMS trigger system.

\section{The CMS Trigger}

\subsection{Overview of the system}

The CMS Trigger system is designed $\left([\because, 5]^{2}\right)$ to provide the output data volume rate at the level of $O(100 \mathrm{MB} / \mathrm{s})$. Each event recorded in the CMS detector results in approximately $1 \mathrm{MB}$ of zero suppressed data. This limits the final event storage rate to about $100 \mathrm{~Hz}$. The event selection at CMS is done in two triggering steps only (Level-1 Trigger, High-Level Trigger).

The Level-1 Trigger is based on custom, partially programmable hardware devices (mostly special-purpose ASICs but also FPGAs where appropriate). The Level-1 Trigger reduces the $40 \mathrm{MHz}$ LHC beam collision rate below $100 \mathrm{kHz}$, which is the maximal input rate for the CMS Data Acquisition (DAQ) and the High-Level Trigger (HLT) system. A schematic view of the central part of CMS DAQ is shown in Figure 11. Data from the detectors are stored in modules of the detector Front-End system upon the accept command is issued by the Level-1 Trigger. Then fragmented data are read out and buffered in Readout Units (RU). The data from one event pass Readout Builder Network to be finally assembled in Builder Unit (BU) and analyzed in the Filter Unit (FU). The BU and FU run in a single commercial PC operated under Linux. Each event is assigned to a single processor where the HLT algorithms are executed and the actual event selection takes place. In the baseline it is planned to have a Filter Farm based on $O(1000)$ double processor FUs. The final treatment of the events selected at HLT is addressed to Computing Services that forward the data to mass storage and perform some monitoring tasks. The DAQ system is completed with the Event Manager, responsible for the data flow control, and Control System which takes care of configuration, DAQ monitoring and various controlling tasks.

\footnotetext{
${ }^{1}$ A start of LHC operation is currently planned for fall 2008.

${ }^{2}$ See [6, 7] for recent updates and current status of the project.
} 


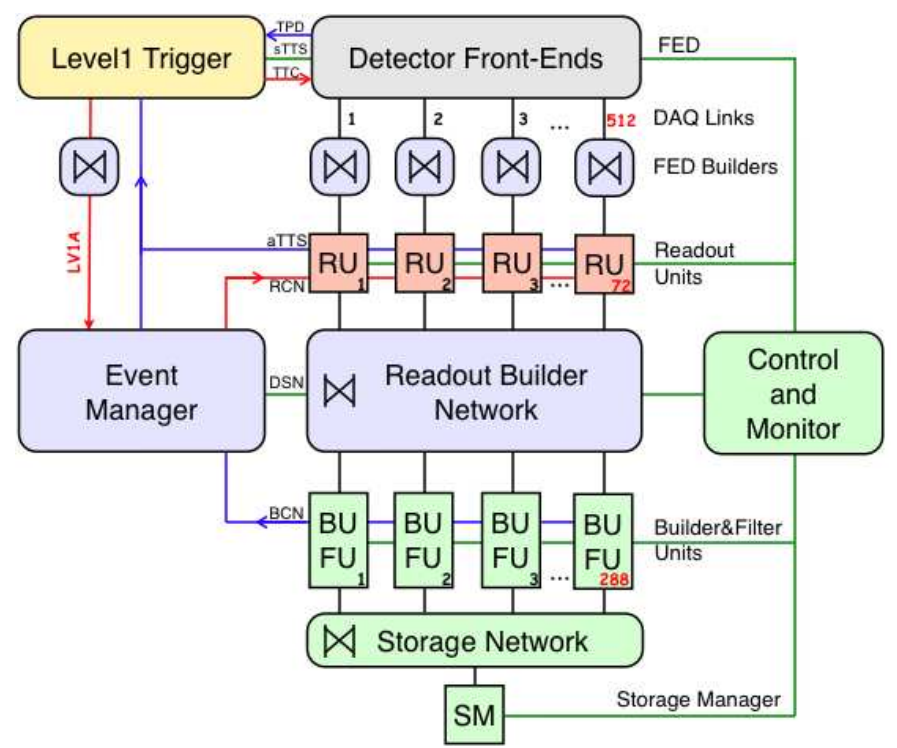

Figure 1: Schematic view of one slice $(72 \mathrm{RU}+248 \mathrm{BU} / \mathrm{FU})$ of the central part of the CMS DAQ

An important feature of the CMS DAQ is that the system is build out of eight $12.5 \mathrm{kHz}$ units. This modularity makes the DAQ system staging possible. It is especially important because the expected initial event rate at LHC will be substantially lower than the design rate. Only a part of the system has therefore to be ready at startup, allowing overall cost optimisation. The CMS choice, called $50 \mathrm{kHz}$ DAQ scenario is to build only four DAQ units for operation in low luminosity mode, providing only half of the design bandwidth.

\subsection{Level-1 Trigger Deliverables}

The Level-1 Trigger is based on coarse-grained trigger data from the muon system and calorimeters, but not from tracker. The trigger algorithms combine candidate objects from calorimeter and muon sub-triggers. The deliverable objects are: muons, electrons/photons (isolated or not), jets (central, forward), taus, total and missing transverse energy, jet multiplicity.

The possible thresholds and trigger object topologies depends on the trigger strategy and allocated DAQ/HLT bandwidth. Initially it is planned to divide the bandwidth in equal parts to different trigger paths. This strategy provides inclusive selection optimal for discoveries. The choice from trigger table for $2 \cdot 10^{33} \mathrm{~cm}^{-2} \mathrm{~s}^{-1}$ luminosity is given in Table 1 .

\subsection{High-Level Trigger}

The HLT algorithms run on a computer Filter Farm, where each event is processed by a single processor. The reconstruction and selection code is written in Object Oriented C++. To cope with the constraints on the event storage resources, the HLT algorithms must achieve a factor $\sim 1000$ reduction on the event rate. The strategy to eliminate unwanted events may explore fast, simplified but not accurate reconstructions or use of minimal amount of precise information. Both ways are used to optimize the event rejection speed although the latter is preferred, in order to keep the HLT reconstruction code as close as possible to that used for the offline reconstruction. The following features are included in the HLT reconstruction code: reconstruction on demand - the 


\begin{tabular}{lcc}
\hline & Threshold $(\mathrm{GeV}, \mathrm{GeV} / \mathrm{c})$ & Individual rate $(\mathrm{kHz})$ \\
\hline Inclusive $e / \gamma$ & 22 & 4.2 \\
Double $e / \gamma$ & 11 & 1.1 \\
Inclusive $\mu$ & 14 & 2.7 \\
Double $\mu$ & 3 & 3.8 \\
Inclusive $\tau$ & 100 & 1.9 \\
Double $\tau$ & 66 & 1.8 \\
\hline
\end{tabular}

Table 1: The selection from Level-1 Trigger table for $2 \cdot 10^{33} \mathrm{~cm}^{-2} \mathrm{~s}^{-1}$ luminosity and $50 \mathrm{kHz}$ DAQ scenario. The CMS trigger thresholds values correspond to $90-95 \%$ efficiency with respect to plateau of efficiency curve. In the case of double triggers both objects have to pass the same, listed, threshold. The cumulative rate of all trigger channels is about $20 \mathrm{kHz}$, (lower than the available bandwidth) due to additional safety factors applied to correct for uncertainties of background treatment. The full trigger table can be found in [8].

reconstruction is started only if it is required by the trigger decision; regional reconstruction data are reconstructed only in the region of interest, defined by a trigger condition; partial and conditional track reconstruction - tracking is stopped if the combinatorial ambiguities are solved and/or the precision is sufficient for further triggering steps or the track fit parameters disagree with the kinematic requirements.

The event reconstruction and selection takes place in several steps. Because the event reconstruction is triggered by Level-1 Trigger based on calorimetry and muon system, the natural way is to fully explore the provided information with full granularity reconstruction therein. The calorimeter and muon system based reconstruction is called Level-2. These reconstructed objects may be supplemented with limited information from the Tracker System (usually by Pixel Detector hits) at Level-2.5 and finally reconstructed using more Tracker data at Level-3. These virtual trigger levels are a matter of convention.

\section{Track and Vertex reconstruction at HLT}

The CMS track reconstruction relies on the tracker detector. It is located in a $4 \mathrm{~T}$ solenoidal magnetic filed. The CMS tracker consists of a silicon Pixel Detector surrounded by a Silicon Strip Tracker.

The Pixel Detector is formed by three barrel layers and two endcap disks on each side. The barrel layers are located at mean radii of 4.4, 7.3 and $10.2 \mathrm{~cm}$ and are $53 \mathrm{~cm}$ long. The two disks are placed at 34.5 and $46.5 \mathrm{~cm}$ from the interaction point. To achieve optimal resolution of the vertex position in the transverse and the longitudinal planes, a design with a rectangular pixel shape of dimensions $150 \times 100 \mu \mathrm{m}^{2}$ was chosen. The hit resolution, enhanced due to charge sharing induced by the large Lorentz drift in the magnetic field, is approximately $10 \mu \mathrm{m}$ in $r-\varphi$ and about $20 \mu \mathrm{m}$ in $r-z$. The whole pixel system consists of about $6.6 \cdot 10^{7}$ pixels in 1400 detector modules.

The Silicon Strip Tracker is a composed detector, organized again in cylindrical layers in barrel and disks in endcap. There are several sensor geometries with the strip lengths varying from 9 to 
$21 \mathrm{~cm}$ and a pitch ranged in 80 to about $200 \mu \mathrm{m}$. The ten barrel layers covers the radius up to $1.1 \mathrm{~m}$. while twelve endcap disks extends to about $2.75 \mathrm{~m}$ along the beam line. Four out of ten layers in the barrel and several rings of endcap disks are equipped with double sided modules tilted by a small angle of $100 \mu \mathrm{rad}$ in order to improve resolution along strip length. The overall size of strip tracker is about $200 \mathrm{~m}^{2}$ of active silicon.

The track reconstruction [5, 9, 10] is started upon of request by previous trigger object and is preceded by the readout of tracker data, its clusterisation and hit reconstruction. The baseline algorithm of track reconstruction in CMS is called Combinatorial Track Finder (CTF) and can be divided into three steps: seed finding - search for sets of hits compatible with desired track kinematics; pattern recognition - collection of all hits compatible with unique track; final fitting reevaluation of trajectory parameters in order to get optimal track parameters.

The key point for fast and robust track reconstruction at HLT is the seeding step. In the baseline it is supported by standalone track and vertex reconstruction in Pixel Detector.

\subsection{Pixel-based reconstruction [11, 12]}

The Pixel detector provides high resolution, three-dimensional space points for precise pattern recognition. With three pixel hits per charged particle, tracks can be approximately reconstructed and primary-vertices can be found. Such a "pixel" reconstruction is useful for track seeding, primary-vertex finding and in a variety of HLT algorithms. The Pixel detector is the most suitable for these tasks due to its good spatial resolution and relatively low occupancy.

Finding Hit Pairs and Hit Triplets The track finding based on pixel hits consists of two steps: definition of a set of hit pairs compatible with a track and from these pairs making a prediction of the third hits. Two precise hits (pairs) are enough to define a track seed, but the ghost rate is high. Track candidates based on three pixel hits (triplets) allow us to do primary-vertex reconstruction and to define simple algorithms for online event selection, even if they are not fully efficient. The search for track candidates based on two pixel hits is performed inside a region of interest with kinematic constraints (Tracking Region). A Tracking Region is defined by a direction, a vertex point from which tracks are expected to originate, a minimal transverse momentum, a maximal transverse impact parameter, and a maximal allowed distance from the vertex along the beam line. In addition, a range of tolerances in the pseudorapidity $(\eta)$ and in the azimuthal angle are considered.

A hit pair consists of two hits in different layers. The analytical constraints from Tracking Region definition has to be fulfilled. In the pseudorapidity coverage of pixel detector a particle is crossing 3 pixel layers resulting in high (above 99.6\%) efficiency ${ }^{3}$ to find a hit pair. The hit pair finding purity is rather low: $1-10 \%$ depending on region size and kinematical constraints. A pairs of hits together with the kinematic constraints from the Tracking Region are used to predict the third compatible hits to form a hit triplets. Hit triplet finding efficiency, in expected detector conditions $^{4}$, is at the level of $85-90 \%$ with corresponding purity $90-99 \%$.

The triplet can be used to determine particle momentum together with longitudinal and transverse impact parameters. Due to poor bending arm the momentum resolution is limited, and de-

\footnotetext{
${ }^{3}$ The hit pair and hit triplet finding efficiency is defined as a number of pairs or triplets associated to track. Only tracks with $|\eta|<2.0$ and transverse momentum above $1 \mathrm{GeV}$ contribute.

${ }^{4}$ The detector geometrical inefficiencies, sensor inefficiencies and readout losses are taken into account.
} 
grades with increasing momentum. Thus for tracks with $p_{T}$ above $10 \mathrm{GeV} / \mathrm{c}$ this measurement is not considered reliable. The example of transverse momentum resolution and longitudinal impact point resolution is given in Figure 2.
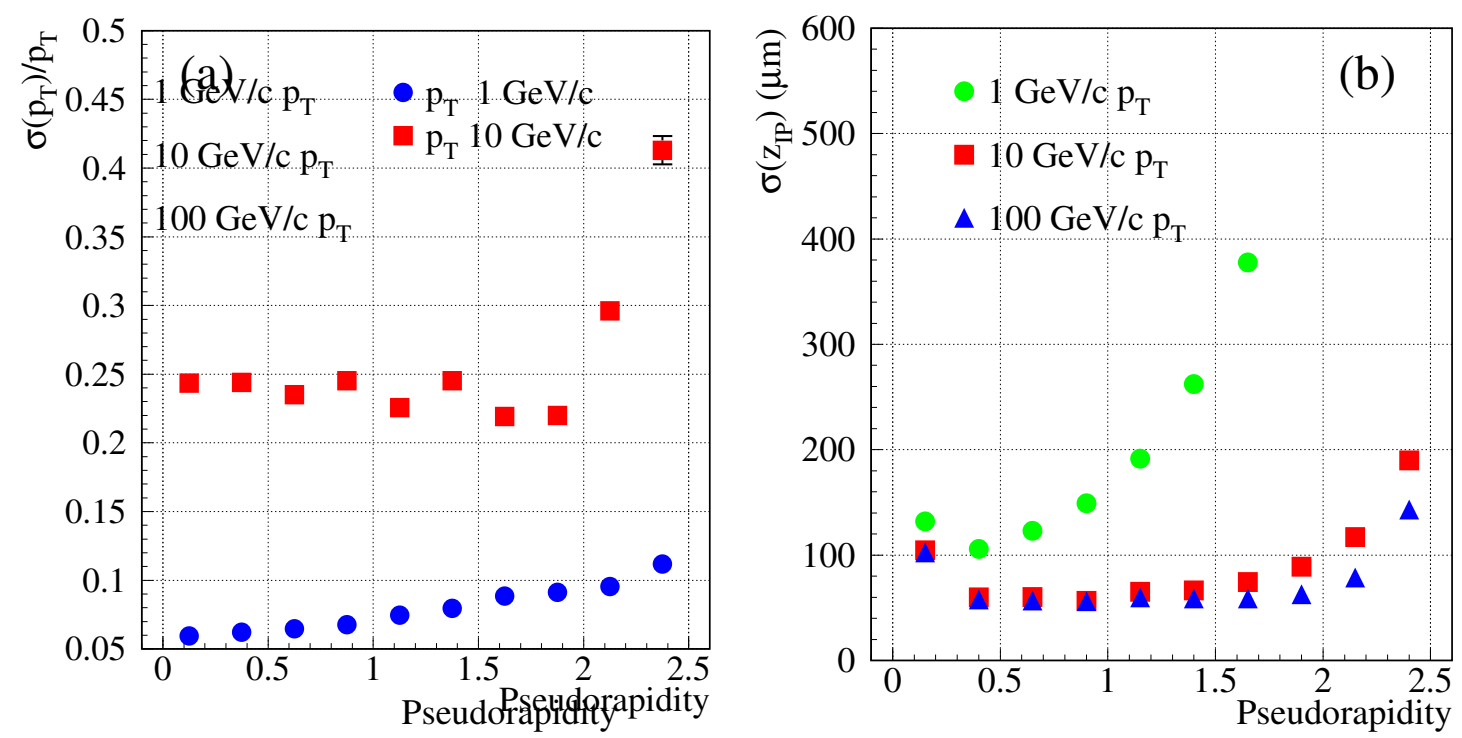

Figure 2: Pixel tracks: A normalised resolution of transverse momentum (a) and impact point along beam axis (b) as a function of pseudorapidity.

Vertex finding Primary-vertex finding based on the pixel hits provides a simple and efficient method for measuring the position of the primary-vertex. This measurement is subsequently used for track seeding and in many HLT analyses. It must therefore be sufficiently accurate and fast. For this reason primary-vertex finding is reduced to a one-dimensional search along the beam $(z)$ axis.

The baseline primary-vertex finding algorithm (called divisive method) make use of the pixel tracks reconstructed from hit triplets. Usually triplets found in the full pixel detector acceptance (global region) are used. However the triplet finding can also be restricted to a selected region in order to make the vertex finding faster. The search for the primary-vertex along the $z$ axis is based on the longitudinal impact point $\left(z_{\mathrm{IP}}\right)$, evaluated from pixel tracks. Only pixel tracks reconstructed with $p_{T}$ in excess of $1 \mathrm{GeV} / c$ and a transverse impact point smaller than $1 \mathrm{~mm}$ are used for primaryvertex finding.

The algorithm looks for large $z_{\text {IP }}$ intervals without tracks to divide the $z$ axis into several groups. An average primary-vertex position is computed from all tracks in each group. Tracks not compatible with that average position are discarded. The discarded tracks are recovered to make a new vertex candidate. This iterative procedure stops when all tracks are found to be compatible with the corresponding primary-vertex positions.

After vertex finding, the vertex candidates are sorted in decreasing order according to the sum of their track $p_{T}^{2}$, with a $p_{T}$ ceiling at $10 \mathrm{GeV} / c$ to account for the imprecision in the $p_{T}$ measurement for three-hit pixel tracks. 
Among the primary-vertex candidates, the closest primary-vertex is defined as that closest in $z$ to the simulated signal primary-vertex, and the tagged primary-vertex as the one with the largest $p_{T}$ sum. The efficiency to find the primary-vertex (tagged $\varepsilon_{\text {tag }}$ or closest $\varepsilon_{\text {close }}$ ) is defined with respect to vertices reconstructed inside a small window around the position of the generated signal primary-vertex. The example efficiencies, for low-luminosity pile-up are given in Table 2 ,

For most event types the primary-vertex is recovered with an efficiency close to $100 \%$. It is significantly lower for events such as $\mathrm{H} \rightarrow \gamma \gamma$, where the low charged-particle multiplicity does not allow the signal primary-vertex to be always distinguished from the pile-up primary-vertices. The primary-vertex $z$ position is reconstructed with a resolution better than $50 \mu \mathrm{m}$ (typically $30 \mu \mathrm{m}$ ).

\begin{tabular}{||l|c|c|}
\hline & $\varepsilon_{\text {close }}$ & $\varepsilon_{\mathrm{tag}}$ \\
\hline $\mathrm{u}$-jets; $E_{T}=10 \mathrm{GeV}$ & 1.00 & 0.99 \\
$\mathrm{u}$-jets; $50<E_{T}<100 \mathrm{GeV}$ & 0.99 & 0.94 \\
$\mathrm{~b}$-jets; $30<E_{T}<50 \mathrm{GeV}$ & 1.00 & 0.96 \\
$\mathrm{H}\left(115 \mathrm{GeV} / c^{2}\right) \rightarrow \gamma \gamma, \mathrm{g}$ fusion & 0.94 & 0.80 \\
$\mathrm{H}\left(150 \mathrm{GeV} / c^{2}\right) \rightarrow \mathrm{ZZ} \rightarrow 2 \mathrm{e} 2 \mu$ & 1.00 & 1.00 \\
$\mathrm{~B}_{s}^{0} \rightarrow \mathrm{J} / \psi \phi$ & 0.97 & 0.78 \\
$\mathrm{t} \overline{\mathrm{t}}$ & 1.00 & 1.00 \\
$\mathrm{t} \overline{\mathrm{t}}, \mathrm{H}\left(120 \mathrm{GeV} / c^{2}\right) \rightarrow \mathrm{b} \overline{\mathrm{b}}$ & 1.00 & 1.00 \\
\hline
\end{tabular}

Table 2: Efficiencies of signal primary-vertex finding at low luminosity. The $\varepsilon_{\text {close }}$ is the efficiency to find correctly a primary-vertex on the list of reconstructed vertices. In the case of $\varepsilon_{\text {tag }}$ the primary-vertex must be also correctly tagged.

\subsection{Performance of Combinatorial Track Finder}

A very important application of described pixel-based reconstruction is seeding of CTF. A seed must provide a rough estimate of track kinematics (trajectory parameters and errors) in order to form initial trajectory candidate necessary for further steps. In the current CMS baseline the seeds are defined be pixel hit pairs, optionally associated to primary-vertex obtained also in pixel reconstruction. The next step in track reconstruction is collection of all hits compatible with unique track (Pattern Recognition). It is an iterative procedure, based on the Kalman filter method. The trajectory candidate (initially given by a seed) is extrapolated to the next tracker layer. The compatible hits are gathered, using $\chi^{2}$ compatibility criteria. The hits provide a new set of trajectory candidates with more precise, updated trajectory parameters (taking into account track bending in magnetic field, multiple scattering and energy losses). One extra trajectory candidate is created with a dummy hit (possessing no position information) ${ }^{5}$. The quality of trajectory candidates is checked and best ones (typically 5, value optimised for low luminosity) are kept for further propagation. The quality filtering is highly configurable and in the standard case takes into account number of all and consecutive dummy hits (1), minimal transverse momentum below which trajectory is rejected $(0.9 \mathrm{GeV} / \mathrm{c})$, maximum $\chi^{2}$ (30) of hits considered compatible with predicted track

\footnotetext{
${ }^{5}$ Dummy hits in trajectory indicate layers where no compatible hits were found.
} 
state. In addition, the ambiguities between tracks sharing the significant fraction (0.5) of hits are resolved. When all hits are collected the trajectory parameters are reevaluated (Final Fitting) in order to get optimal track parameters. It is least-squares fit in the form of Kalman filter. It consists of "forward" (from beam-line out) and "backward" (towards beam-line) fits providing best estimation of parameters at the end and at the beginning of trajectory respectively, and - combined - for each intermediate layer. At this step the hit positions are recomputed with improved incident trajectory estimates. Possible biases included in initial seed kinematics are removed.

The full track reconstruction with Combinatorial Track Finder leads to much better resolution comparing with pixel-based reconstruction. The example performance for single muon case is presented in Figureß.
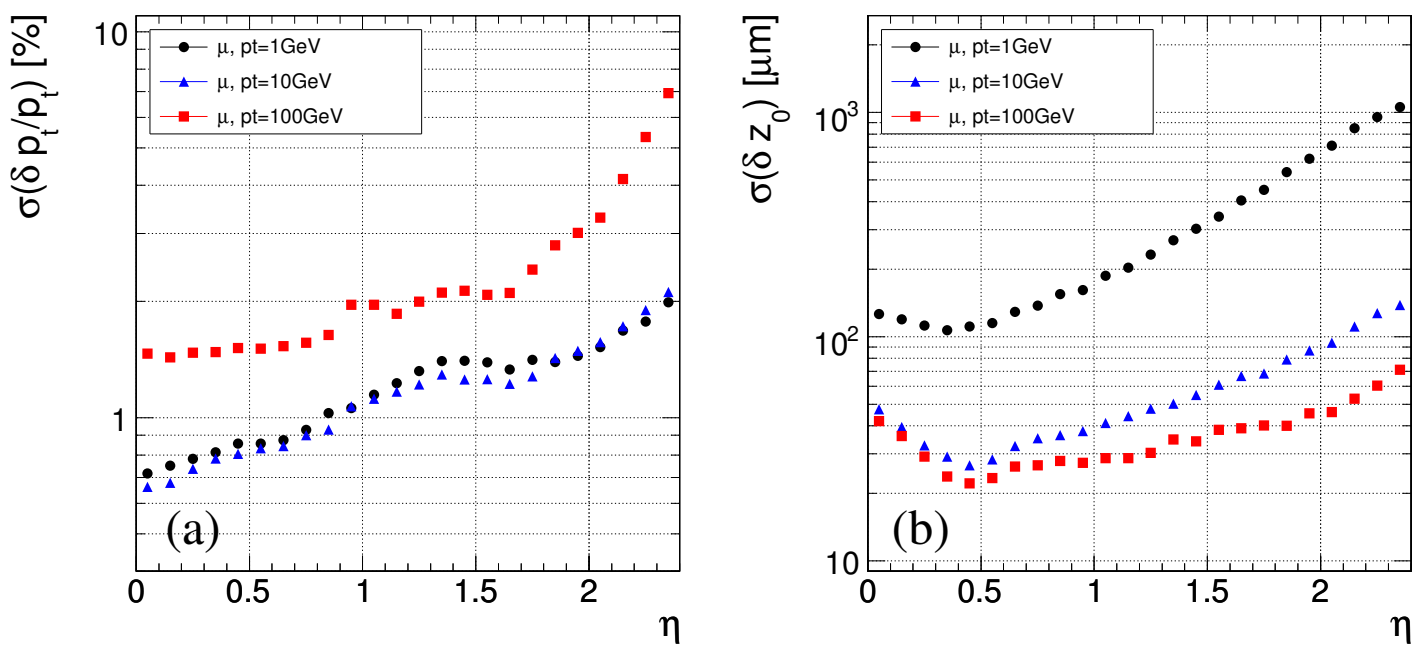

Figure 3: CTF tracks: A normalised resolution of transverse momentum (a) and impact parameter along beam axis (b) as a function of pseudorapidity.

\section{Track based algorithms in HLT}

The track based algorithms are widely used [13]-[18] in the CMS High-Level Trigger for a object reconstruction and isolation. An interesting example is a muon reconstruction.

The HLT muon reconstruction is divided into standalone reconstruction in muon system (Level2) and combined muon system-tracker reconstruction (Level-3). The reconstruction is seeded by and Level-1 muon candidates, which in most cases are given by real muons with mismeasured momentum. The Level-2 muon algorithm works in a region of interest defined by the Level-1. It uses locally reconstructed muon chamber information. The muon trajectory is built and updated by collecting measurements using the Kalman Filter fit in muon system. Since the most interesting muons originate from the beam spot or nearby, the momentum measurement is improved by updating the muon state with the vertex constraint.

In the baseline, the Level-3 muon reconstruction starts with the determination of a Tracking Region, with parameters and uncertainties defined by the Level-2 muon state at the vertex. The 
seeds for the regional reconstruction are given by Pixel hit pairs and the standard Combinatorial Track Finder tracks are reconstructed. The ones compatible with initial Level-2 muon are then updated with muon measurements and form final Muon track. The typical momentum resolution of $O(10 \%)$ at Level-2 is improved to $O(1 \%)$ at Level-3 (see Figure 4 ).
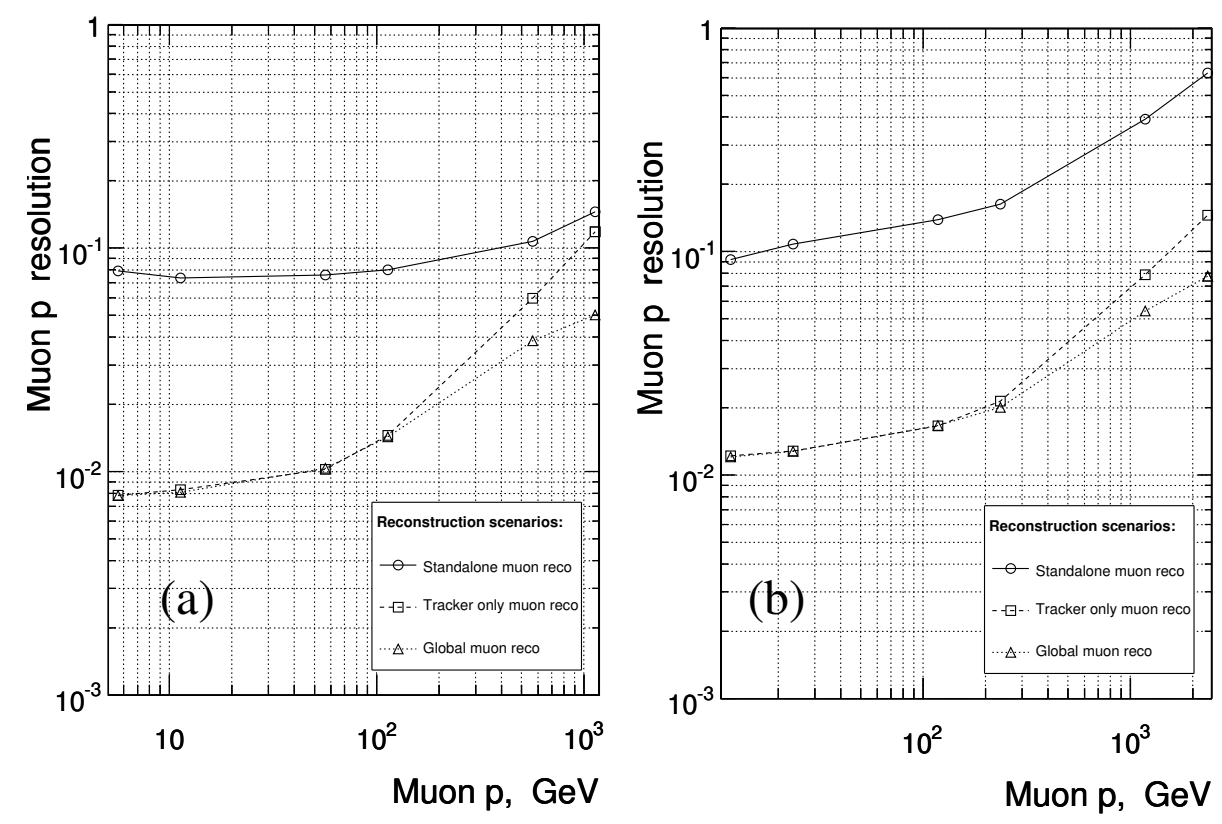

Figure 4: The normalized momentum resolution (sigma of Gaussian fit to $\frac{q^{r e c} / p^{r e c}-q^{g e n} / p^{g e n}}{q^{g e n} / p^{g e n}}$, where $q^{r e c, g e n}, p^{r e c, g e n}$ - reconstructed and simulated muon charge and momentum) as a function of particle momentum for barrel ( $\eta=0.5$, (a)-left plot) and endcaps ( $\eta=1.5$, (b)-right plot). The performance of standalone muon track, tracker only, and combined fit (global muon reco) is shown.

The muon rate in the $p_{T}$ range below $30 \mathrm{GeV} / c$ is dominated by muons from $b, c$ and $K, \pi$ decays. In order to separate them from those produced in heavy object decays, isolation algorithms are used (Figure 5 ). Three techniques were studied: calorimeter isolation, which combines calorimeter objects, pixel isolation, with tracks from pixel reconstruction and tracker isolation, with tracks reconstructed by standard regional reconstruction algorithm. The isolation algorithms apply a threshold (which may depend on $\eta$ ) to the deposits $\left(\sum E_{T}\right.$ or $\left.\sum p_{T}\right)$ in a cone around the muon. The performance of pixel isolation and tracker isolation is comparable. A good charged particle track measurement in tracker and measurement of neutral particle energy in calorimeters made pixel/tracker and calorimeter isolation complementary.

\section{Summary}

The CMS High-Level Trigger has to reduce maximal Level-1 output rate of $100 \mathrm{kHz}$ rate to $O(100) \mathrm{Hz}$ which is feasible for mass storage. The High-Level Trigger is based on farm of commercial processors where algorithms are executed. The track finding plays a important role for most of HLT algorithms. A Combinatorial Track Finder - baseline CMS tracking algorithm provides high quality reconstructed tracks. It is seeded with results of standalone reconstruction in Pixel Detector. 

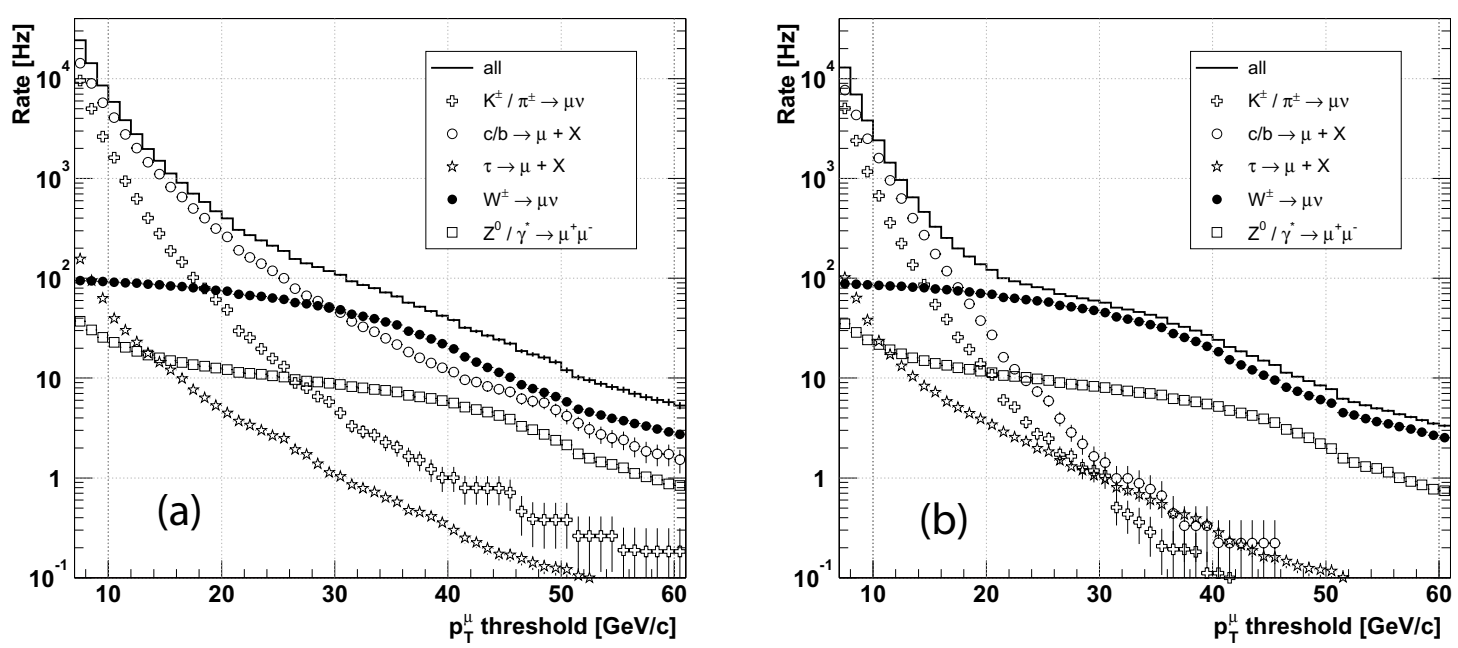

Figure 5: Muon rates before (a) and after (b) isolation. The $b, c$ and $K, \pi$ contribution is suppressed. The plot is made for $10^{34} \mathrm{~cm}^{-2} \mathrm{~s}^{-1}$ to better pronounce the isolation effect. The efficiency loss for a typical signal (like $W \rightarrow \mu v$ on the plot) is approximately $7 \%$ for all isolation algorithms applied in cascade.

The pixel-based reconstruction provides not only pairs of hits suitable for seeding but also list of primary-vertices and hit triplet tracks. Pixel tracks are widely used by HLT algorithms were high efficiency is not mandatory, like isolation.

\section{References}

[1] C. Jarlskog and G. Rein, Proceedings of the Large Hadron Collider Workshop, Aachen, 4-9 October 1990, CERN 90-10, ECFA 90-133

[2] CMS Collaboration, CMS, The Compact Muon Solenoid, Letter of Intent, CERN/LHCC 92-3

[3] CMS Collaboration, CMS, The Compact Muon Solenoid, Technical Proposal, CERN/LHCC 94-38

[4] CMS Collaboration, CMS, The Trigger and Data Acquisition project, Volume I: The Level-1 Trigger, CERN/LHCC 2000-038

[5] CMS Collaboration CMS, The Trigger and Data Acquisition project, Volume II: Data Acquisition \& High-Level Trigger, CERN/LHCC 2002-26

[6] A. Afaq et al, The CMS High Level System, CMS CR 2007/017

[7] G. Bauer et al, CMS DAQ Event Builder Based on Gigabit Ethernet, CMS CR 2007/017

[8] CMS Collaboration, CMS Technical Design Report, Volume II: Physics Performance, CERN/LHCC 2006-021, J.Phys.G34:995-1579, 2007.

[9] CMS Collaboration CMS Technical Design Report, Volume I: Detector Performance and Software, CERN/LHCC 2006-001

[10] W. Adam et al, Track reconstruction in the CMS tracker, CMS NOTE 2006/041

[11] S. Cucciarelli et al, Track-Parameter Evaluation and Primary-Vertex Finding with the Pixel Detector, CMS NOTE 2003/026 
[12] S. Cucciarelli et al, Track reconstruction, primary vertex fi nding and seed generation with the Pixel Detector, CMS NOTE 2006/026

[13] N. Amapane, M. Fierro, M. Konecki, High Level Trigger Algorithms for Muon Isolation, CMS NOTE $2002 / 040$

[14] M. Vos, F. Palla, b-tagging in the High Level Trigger, CMS NOTE 2006/030

[15] S. Gennai em et al, Tau jet reconstruction and tagging at High Level Trigger and off-line, CMS NOTE 2006/035

[16] S. Baffioni et al Electron reconstruction in CMS, CMS NOTE 2006/040

[17] L. Agositno, M. Pieri, HLT Selection of Electrons and Photons, CMS NOTE 2006/078

[18] N. Amapane, The CMS Muon Trigger System, CMS CR 2003/047 